\title{
Towards Planning for Elastic Objects
}

\author{
Lydia E. Kavraki Department of Computer Science, Rice University, Houston, TX 77005 \\ Florent Lamiraux Department of Computer Science, Rice University, Houston, TX 77005 \\ Christopher Holleman Department of Computer Science, Rice University, Houston, TX 77005
}

This paper investigates the problem of path planning for a thin elastic plate. The underlying geometric model for the plate is provided by a Bézier representation. The geometric model is augmented by a realistic mechanical model. The latter permits the computation of the shape of the plate with respect to a set of grasping constraints by minimizing the elastic energy of the deformation. We use a probabilistic roadmap planner to compute paths for the plate and we present a number of experimental results to illustrate our approach. Our work is a first step towards considering the physical properties of objects when planning.

\section{Introduction}

The problem of planning a path for a robot consisting of rigid parts has been studied extensively over the last decade $[17,21]$. This paper describes a first step in the direction of solving a variant of the above problem, namely the problem of planning a path for a flexible robot/part. We focus on the case of an elastic metal plate to illustrate our approach and explore some of the issues arising when considering flexible parts.

Several important applications motivate our research: in industrial settings there is a need to manipulate sheets of metal [26], pipes that can bend [31], and cables [25]. In assembly maintainability studies done with virtual prototyping, planning is used to remove a part from an assembly given only the CAD model of the assembly [10]. The flexibility of the part needs to be considered as engineers use deformable parts to produce compact assemblies [9]. Last but not least, our work may have applications in domains like computer generated animation, where physical properties can help generate realistic sequences, and virtual envi-

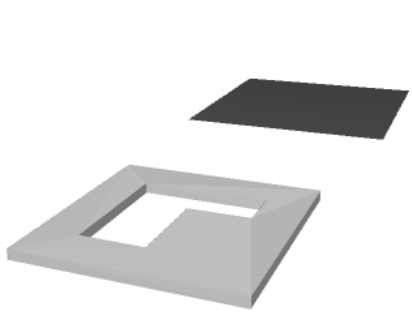

(1)

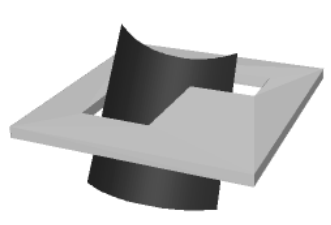

(3)

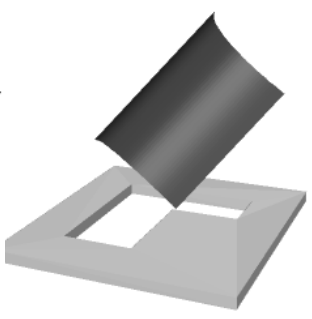

$(2)$

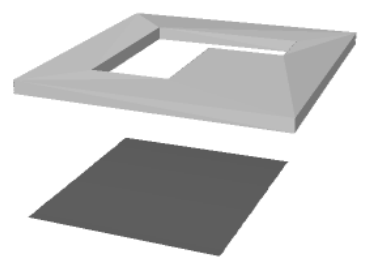

(4)
Figure 1: Snapshots along a path computed by f-PRM for an elastic metal plate that can only bend.

ronments for surgical training, where flexible medical tools are guided inside the human body.

This paper addresses the problem of planning paths among obstacles for a thin elastic metal plate. We assume that the plate is manipulated by two wide grippers that can bend the plate. Although our work is not restricted to manipulation that induces only bending, we focus on this case to discuss our approach in detail. The geometry of the plate is modeled using the Bézier representation, while deformations corresponding to grasping conditions are computed by minimizing the elastic energy of the plate. The elasticity limit of the material of the plate is taken into account and 
we reject deformations that may cause a permanent change in its shape. The actual robots performing the manipulation are not considered. Work in a similar spirit is done in assembly sequencing, where removal paths for parts are computed without taking into account the tools needed to perform the removal [10,39].

We plan paths for the elastic plate using an extension of the Probabilistic RoadMap planner (PRM) in $[19,20,28,33]$. PRM was chosen because it is one of the few planners that can deal efficiently with high dimensional planning problems [20]. The geometric representation of deformations requires in general a large number of parameters and these specify degrees of freedom (dof) for the surface. We call our new planner flexible-PRM (f-PRM). f-PRM builds a roadmap in the configuration space of the plate. The nodes of the roadmap are computed by first selecting random values for the parameters that specify the manipulation constraints and then obtaining an equilibrium configuration for these values. The edges of the roadmap are low-energy paths computed by a local planner. Once a sizable roadmap has been produced, f-PRM attempts to answer a user query by connecting the initial and the final configurations to the roadmap and then searching the roadmap for a sequence of paths between these nodes. A few snapshots along a path produced by fPRM for a surface patch that can only bend (a 7-dof problem) are given in Figure 1.

Although in this paper we concentrate on the case of an elastic plate which is represented by a Bézier surface and can only bend, our approach is not restricted to the specific energy model, geometric representation, and grasping constraints used. As a proof of concept, we use our framework to plan paths for a surface patch that can assume a wide range of deformations due to less restrictive energy and manipulation constraints. This case, which is separately investigated in [18], may be of interest in computer generated animation. We report experiments solving a 27-dof planning problem with few modifications of our original planner.

Our work blends ideas from planning for highdimensional problems (PRM) with work in geometric modeling (representations of curves and surfaces) and mechanics/physics (energy models for elasticity or other physical properties). The randomized framework of f-PRM provides an excellent testbed for investigating several novel issues that arise in the context of planning with flexible objects. These include (a) understanding the implications of using different geometric representations for the objects, (b) acquiring meaningful energy models, (c) studying manipulation of flexible objects and its effect on the attainable deformations of these objects, (d) devising algorithms for planning low-energy paths between configurations with different deformations, (e) investigating approaches to efficient collision checking when the shape of the object changes, and (f) developing methods for improving the overall quality of the paths.

This paper is organized as follows. Section 2 briefly surveys related work. Section 3 addresses geometric and mechanical issues related to the representation of an elastic plate and its manipulation. Section 4 outlines the general principles of f-PRM. We present in Section 5 experimental results for an elastic surface that can only bend. We discuss in Section 6 how to use f-PRM for planning with a less restrictive energy model.

\section{Related Work}

Path planning for a flexible surface defines a highdimensional problem and fortunately much progress has been made in robotics on this front. However little has been done to accommodate the physical properties of robots such as their elasticity. Issues related to object deformation have been studied mostly in the areas of mechanics, geometric modeling, and graphics. We briefly survey separately each of the above areas.

Robotics Recent work on the path planning problem has produced several practical planners for robots that consist of rigid parts (see for example [1, 2, 4, $11,16,20])$. These methods routinely take into account geometric constraints such as joint limits and obstacles, but also constraints arising from kinematics such as nonholonomic velocity constraints due to the rolling without slipping of wheeled mobile robots [22], or constraints over the radius of curvature of a car-like system [23, 33]. 
With the exception of the areas of dynamics and control that have guided the design of modern robots [30], there are few cases where physical constraints and planning have been tightly coupled. Kino-dynamic motion planning is one such case as it takes into account dynamic constraints by planning in the tangent space of the configuration space $[12,13]$.

As far as deformable robots and parts are concerned, a lot of work has been done in the context of manipulation. Robots with flexible links are now being built since they facilitate certain tasks (like hammering a peg into a hole) and their modeling and control is under development (for pointers to current work see [26]). Recent papers consider the dynamic analysis of robots with flexible payloads such as two robots manipulating a flexible metal sheet [26] or a vibrating object [31], or solve the task of inserting one end of a flexible wire into a hole while holding the other end [25]. Furthermore, research in snake-like robots has explored issues related to "geometric mechanics" that are relevant to our discussion [7, 27]. For example, the work [27] describes the net motion of a snake robot as a function of variations in the mechanism's shape variables.

Mechanics In mechanics there exists a large amount of research on modeling physical properties such as elasticity [6]. The work in [38] discusses the case of thin plates and develops an energy model for the deformation of a thin elastic plate that depends only on the planar deformation and the curvature of the plate.

Geometric modeling In geometric modeling several representations for curves and surfaces have been developed to enable accurate manipulation of shape while considering a relatively small number of parameters $[5,14]$. In this paper we use a simple model from computer-aided geometric design for our flexible surfaces, namely the tensor product Bézier surface. Other models may also be appropriate and this issue is currently under investigation.

Graphics In graphics physically based models have been proposed for deformable parts $[34,35]$. The use of physical simulation and related optimization techniques as a means of geometric interaction has been applied to animation [36], drawing [37], free-form sur- face and volume modeling [8], mechanical design [40], and interactive molecular simulation [32]. For a discussion on the dynamic simulation of non-penetrating flexible bodies see [3].

\section{Deformation of an Elastic Plate}

Before considering planning, we present the geometric and mechanical model used for the elastic plate. We assume that the plate is manipulated by two robot grippers. In this paper we do not consider the full version of the manipulation problem: we only specify conditions, which we call grasping constraints or limit conditions, and which reflect the effect of manipulation on our object.

Let $F_{A}=\left(\vec{w}_{1}, \vec{w}_{2}, \vec{w}_{3}\right)$ be an orthonormal reference frame for the undeformed configuration of the plate, which we also refer to as the load-free configuration. In that configuration the plate occupies the volume

$$
\left\{\begin{array}{l}
0 \leq x_{1} \leq L \\
-\frac{W}{2} \leq x_{2} \leq \frac{W}{2} \\
-\frac{h}{2} \leq x_{3} \leq \frac{h}{2}
\end{array}\right.
$$

We assume that $h$ is small compared to $L$ and $W$ and we represent the geometry of the plate by a surface. However, we take into account the thickness $h$ of the plate in all energy calculations.

\subsection{Geometric model for the plate}

Tensor product Bézier surfaces are used to represent our plates. The main advantage in choosing the Bézier representation is that several grasping constraints can easily be specified with the Bézier representation as discussed in Section 3.2.

A Bézier surface is defined by a $n \times m$ control net, made up of a grid of points in 3 -space. Let $P_{i j}, i=$ $0, \ldots, n, j=0, \ldots, m$ be these points, also called the control points or Bézier points. The tensor product approach obtains a point on the surface as

$$
S(u, v)=\sum_{i=0}^{n} \sum_{j=0}^{m} B_{n}^{i}(u) B_{m}^{j}(v) P_{i j}
$$




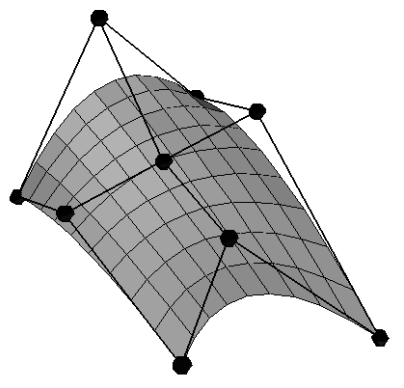

Figure 2: A biquadratic Bézier surface patch with its 9 control points and several isolines.

where $u \in[0,1], v \in[0,1]$, and $B_{n}^{i}(u), B_{m}^{j}(v)$ are the Bernstein polynomials defined by

$$
B_{n}^{i}(u)=\left(\begin{array}{c}
n \\
i
\end{array}\right) u^{i}(1-u)^{n-i} .
$$

In the following, we will denote by $t_{u}=\partial S / \partial u$ and $t_{v}=\partial S / \partial v$ the tangent vectors to the surface. In the load free configuration, the control points have the following coordinates $P_{i j}=\left(\frac{i}{n} L, \frac{j}{m} W, 0\right)$.

The surface contains the four corner control points. The rest of the control points are not necessarily on the surface but affect its shape. A simple illustration of a biquadratic surface is given in Figure 2 .

\subsection{Grasping constraints for manipulation}

We consider now a plate made of an elastic material and we act on it by grasping two opposite edges using for instance two wide grippers. Let us assume that we can control the distance $d<L$ between these parallel grippers as shown on Figure 3 . The elasticity hypothesis states that there exists a mapping from the space of deformations of the plate into $\mathbf{R}_{+}$called the elastic energy of the plate. An equilibrium deformation is a local minimum of the elastic energy in the space of deformations fitting the limit conditions imposed by a given grasping. We call simple bending the set of deformations reachable by these grasping conditions. Simple bending induces deformations for which the tangent vector $t_{v}$ is constant over the surface and equal to its value in the undeformed configuration. Any deformation can then be obtained from the curve $S(u, 0)$ by

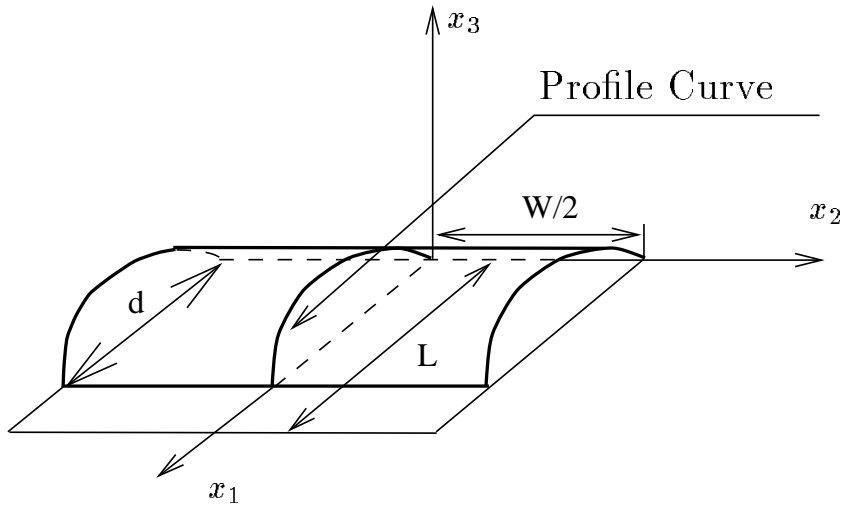

Figure 3: Grasping by two opposite parallel edges.

the following translation

$$
S(u, v)=S(u, 0)+\left(v-\frac{1}{2}\right) W \vec{w}_{2} .
$$

We can thus represent this deformation by a Bézier curve in the $\left(\vec{w}_{1}, \vec{w}_{3}\right)$-plane. That is

$$
S(u)=\sum_{i=0}^{n} B_{n}^{i}(u) P_{i},
$$

with $u \in[0,1]$. We call this curve the profile curve of the surface (see Figure 3). The grasping constraint implies $S(0)=P_{0}, S(1)=P_{n}$. The position of the other control points is computed by minimizing the elastic energy of the plate as defined in Section 3.4.

\subsection{Geometric analysis of deformations}

We now define the deformation coefficients appearing in the expression of elastic energy. Our discussion draws from [38] which shows that the deformation of a thin elastic plate depends only on the planar deformation and the curvature of the plate. For a general deformation, 6 coefficients are required: 3 for the planar strain and 3 for the curvature. Under simple bending, however, we need only consider the deformations of a curve. In this case, all but one of the strain coefficients and one of the curvature coefficients are zero. We analyze below the simple bending case and provide some intuition for the meaning of the coefficients involved in the expression for elastic energy. We treat the general 2D case in the Appendix. 
Let us consider the profile curve $S(u)$ as shown in Figure 3 . In the load free configuration, the position of the control point $P_{i}$ is $(i L / n, 0,0)$ and the corresponding Bézier curve is given by $S(u)=(L u, 0,0)$. The tangent vector $t_{u}(u)=S^{\prime}(u)$ to this curve is thus constant and equal to $(L, 0,0)$. As shown in Figure 4, a deformation can be defined by an extension coefficient $\varepsilon$ and a curvature $\chi$. The expressions of these coefficients are

$$
\begin{aligned}
& \varepsilon(u)=\frac{1}{L}\left(\left\|t_{u}(u)\right\|-L\right), \\
& \chi(u)=\frac{\left\|t_{u}(u) \times t_{u}^{\prime}(u)\right\|}{\left\|t_{u}(u)\right\|^{3}},
\end{aligned}
$$

where $t_{u}^{\prime}(u)=S^{\prime \prime}(u)$ is the derivative of $t_{u}(u)$ in the deformed configuration and $\times$ is the cross product. It can easily be seen that $\varepsilon$ represents the extension rate of a small piece of matter of length $d l_{0}$ in the $\vec{w}_{1}$ direction. That is $\varepsilon=\left(d l-d l_{0}\right) / d l_{0}$, where $d l$ is the length of the small piece of matter after deformation (see Figure 4(b)). Equation (3) is the standard formula for curvature, while Figure 4(c) points out that bending the plate induces extension and compression above and below the medium surface. From now on, we will denote the strain vector by

$$
\mathbf{e}(u)=(\varepsilon(u), \chi(u))
$$

\subsection{Mechanical analysis of deformations}

Elasticity theory asserts that for a general surface the elastic energy of a deformation is obtained by integrating the elastic energy per unit of surface $\psi(\mathbf{e}(x))$ over the surface, where $\mathbf{e}(x)$ is the previously defined strain vector at some point $x$ of the surface.

Under our hypothesis of one-dimensional deformation, the elastic energy per unit of surface w.r.t. the local deformation of the plate is given by [38]

$$
\psi(\mathbf{e})=\psi^{\chi}(\chi)+\psi^{\varepsilon}(\varepsilon)
$$

where

$$
\begin{aligned}
\psi^{\chi}(\chi) & =\frac{E h^{3}}{24} \frac{\chi^{2}}{1-\nu^{2}} \\
\psi^{\varepsilon}(\varepsilon) & =\frac{E h}{2} \frac{\varepsilon^{2}}{1-\nu^{2}}
\end{aligned}
$$

(a)

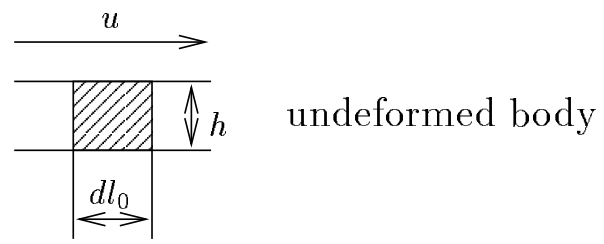

(b)

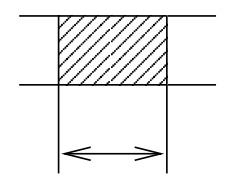

pure extension

$$
\chi=0
$$

(c)

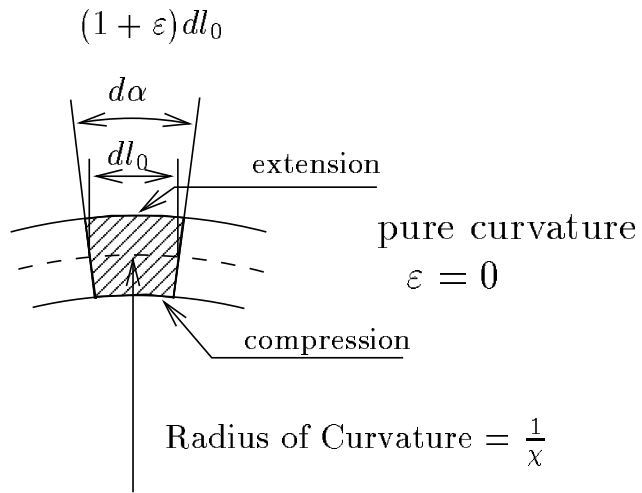

Figure 4: Extension and curvature coefficients.

$E$ and $\nu$ are coefficients characterizing the elasticity of the material ${ }^{1}$. They are respectively called the Young Modulus and Poisson ratio.

\subsection{Equilibrium deformations}

An equilibrium deformation is a local minimum of the elastic energy among the deformations fitting the limit conditions imposed by grasping and thus can be computed by minimizing the elastic energy over the free parameters of the geometric representation of the part.

In the simple bending case, the grasping condition determines the position of two control points of the profile curve: $P_{0}$ and $P_{n}$. Hence, we are required to minimize the elastic energy over the $n-1$ free control points. To perform this minimization, we use the conjugate gradient method [29]. The gradient of the elastic energy is obtained by integrating over the plate the partial derivatives of (4) with respect to the free

\footnotetext{
${ }^{1}$ For instance, for aluminum, $E=74 \mathrm{MPa}$ and $\nu=0.34$.
} 


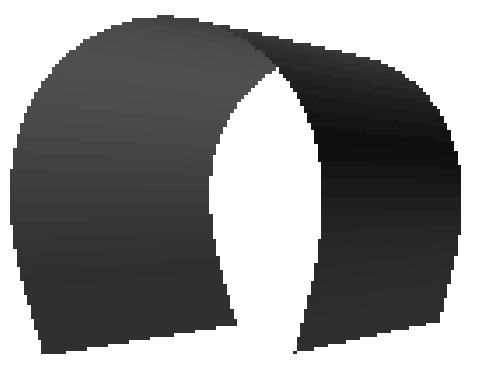

Figure 5: An equilibrium deformation of a surface with a profile curve of 10 control points.

control points. Integrals are computed using the Simpson formula [29].

Figure 5 shows the result of the minimization of the elastic energy for a user-specified $d$, under the simple bending condition and with a profile curve of 10 control points. Notice that the behavior of the surface is the expected one and that the symmetrical surface with respect to the plane defined by the grasping edges is also an equilibrium configuration.

\subsection{Elasticity limit}

The elastic model we use is valid only for small deformations. Out of the elasticity domain, the material has an elasto-plastic behavior. That is, the unconstrained shape of the object is different before and after the deformation and the material is irreversibly affected. We need to avoid this behavior when planning and ensure that all deformations lie in the elasticity domain. We explain now how to express the latter condition in terms of the coefficients $\varepsilon$ and $\chi$ of the strain vector.

Figure 4(c) shows how the curvature of the medium surface implies compression and extension within the thickness of the plate. Thus, for a curvature $\chi$ and an extension $\varepsilon=0$, the maximal strain occurs on the upper and lower surfaces. The straight line of undeformed length $d l_{0}=R d \alpha$ is extended on the upper surface to $(R+h / 2) d \alpha$, where $R=1 / \chi$ is the radius of curvature. Thus, the extension rate is equal to $\chi h / 2$ on the upper surface, and to $-\chi h / 2$ on the lower surface. If $\varepsilon$ is not zero, the extension rate on the upper and lower surface are equal to the sum of $\varepsilon$ and these latter values. (This is a first order approximation [38]).

Therefore, the condition under which all particles of the plate remain in the elasticity domain is

$$
|\varepsilon|+\frac{h}{2}|\chi|<\varepsilon_{\max },
$$

where $\varepsilon_{\max }$ is a constant depending on the material.

In our application, this constraint corresponds to bounding from below the value of the bending parameter $d$

$$
d_{\min }<d<L .
$$

For most metals, $\varepsilon_{\max }$ is very small, i.e. $<10^{-3}$. Note however that if the plate is very thin, a large curvature can occur, as can be seen from equation (7) and illustrated in Figure 5. As a final remark, let us point out that to determine precisely the forces and strain within a material, we would need high precision models (possibly finite elements with hundreds of points). However, for the purposes of our work it is enough to reasonably approximate the shape of the surface and our model achieves this approximation.

\section{Planning: Description of f-PRM}

Our planning method, f-PRM, extends many of the ideas of the probabilistic roadmap approach to planning $[19,20,33]$. The planner repeats a basic step until a query is answered or until an allowed amount of time has elapsed. The method can be seen as a single shot method (answering a single user query), or as a method that consists of a preprocessing and a query processing phase. In both cases, the approach is incremental: the roadmap constructed in the free part $F$ of the configuration space can always be augmented by adding more nodes and more paths, hopefully capturing in greater detail the connectivity of $F$ and allowing for a larger set of user queries to be answered.

Preprocessing: f-PRM constructs a roadmap $R=$ $(V, E)$ in $F$ by repeating the basic step shown in Figure 6. A few comments are in order. 


\section{Preprocessing (basic step):}

1. Generate a low-energy deformation uniformly at random in $F$. Then generate $N$ random configurations having this deformation.

2. Update the graph $R=(V, E)$ in which $E$ consists of connections found by a local planner. Each node is tried for connection with its $K$ closest neighbors among all nodes generated in $\mathrm{V}$.

3. Identify "difficult" areas and refine sampling in these areas by generating $M$ more nodes. Connect the new nodes to $R$ and update $R$.

Figure 6: Roadmap construction.

- At each basic step many configurations with the same deformation are generated. This allows us to create (and later search) for paths that do not unnecessarily deform the plate.

- During step (2) each node $c$ is tried for connection with its $K$ closest neighbors among the nodes in $\mathrm{V}$. These neighbors may be of the same or different deformation as $c$. To find the neighbors, f-PRM uses a distance metric $D(\cdot, \cdot)$ that takes into account the rigid transformations and deformations of the configurations given as its arguments. Using $K$ makes the running time of the algorithm linear in the number of generated nodes. A simple and deterministic local planner is used for the interconnections (see discussion in [20]).

- During step (3), which is also called the node enhancement step, f-PRM identifies difficult areas using the following heuristic scheme: a configuration $c$ is chosen from $V$ with probability

$$
\operatorname{Pr}(c \text { is selected })=w(c),
$$

and a random configuration is generated in the neighborhood of $c$. The weights $w(\cdot)$ used above are chosen as in [19]

$$
w(c)=\frac{1}{d_{c}+1} / \sum_{t=1}^{N} \frac{1}{d_{t}+1}
$$

where $d_{c}$ is the degree of node $c$, that is the number of connections that $c$ has with other nodes. To generate a random node in the neighborhood of $c$, we proceed as follows: we start a random walk from node $c$ and perform a predefined number of reflections. For each reflection, we generate a random direction and move in that direction until the robot collides with an obstacle, or a predefined number of steps have been executed. The end configuration of the random walk is added to $R$ and is tried for connection with its neighboring configurations of any deformation in $R$.

Query processing: given $R$, a starting configuration $s$, and a goal configuration $g$, f-PRM attempts to connect $s$ and $g$ to two close-by nodes $s^{\prime}$ and $g^{\prime}$ that belong to the same connected component of $R$. If successful, the component is searched for a sequence of edges from $s^{\prime}$ to $g^{\prime}$.

\section{Planning in the Presence of Manipu- lation Constraints}

Using the elasticity model for a thin plate specified in Section 3, we proceed to define and solve planning problems under the simple bending hypothesis. We first outline our implementation choices for f-PRM and then describe our experiments.

\subsection{Implementation choices}

Generation of random configurations: the representation of a configuration is broken down into two parts (a) the deformation and (b) the rigid body transformation. The deformation is specified by the coordinates of the control points in the reference frame $F_{A}$ of the load-free configuration. The rigid body transformation is given by a translation and a rotation vector. Decoupling deformations and rigid body transformations allows using the same shape at different positions in the workspace and facilitates collision checking as discussed below.

In the case of simple bending it is enough to specify $d \in\left[d_{\text {min }}, L\right]$ to obtain a deformation. In our current implementation, we discretize the interval of possible values of $d$ into 200 different values. For each value, we 
precompute the corresponding deformation of the profile curve by minimizing the elastic energy. The surface is obtained from the profile curve as specified in Section 3.2. We choose a deformation randomly among the 200 generated off-line. A random rigid body transformation is applied to the deformation. The discretization of $d$ was done to speed up computations and is by no means restrictive.

Collision checking: collision checking is implemented with the RAPID library [24]. This library takes as input collections of triangles describing the environment and the moving object. In our implementation, the plate is approximated by a grid of points evenly sampled across the surface. These points define triangles that are used by RAPID. The obstacles are also decomposed into triangle soups. Once an internal model of the surface and a model of the obstacles have been created by RAPID, a configuration can be queried for collision by specifying a rigid transformation for both models. The creation of an internal model of the surface is expensive compared to the actual collision checks. By keeping the shape of the surface separate from its location in the workspace, the internal model for any deformation can be built once and reused, speeding up collision checking.

Distance measure: let $c_{1}, c_{2}$ be two configurations of the surface. Let $P_{i}^{c_{1}}, P_{i}^{c_{2}}$ denote the positions of their control points, $s_{1}, s_{2}$, the translation vectors of their rigid transformations and $\theta$ the rotation angle to obtain $c_{2}$ from $c_{1}$. The distance $D$ of $c_{1}$ and $c_{2}$ is given by

$D\left(c_{1}, c_{2}\right)=\left\|s_{1}-s_{2}\right\|+|\theta| l_{\max }+\max _{i}\left\{\left\|P_{i}^{c_{1}}-P_{i}^{c_{2}}\right\|\right\}$.

In the above, $l_{\max }$ denotes half of the diagonal length of the undeformed plate. This term approximates the greatest extent of the surface (see [4] for handling rotation).

Local planner: to connect $c_{1}$ and $c_{2}$ the local planner first finds the axis of rotation and angle of rotation needed to get from one configuration to the other. Then a path is constructed by linear interpolation of the translation and rotation vector between $c_{1}$ and $c_{2}$.

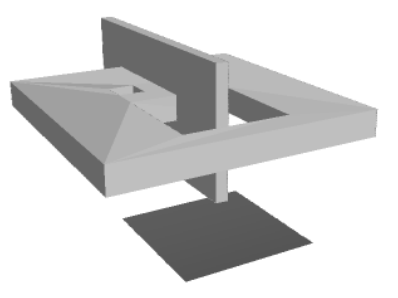

(1)

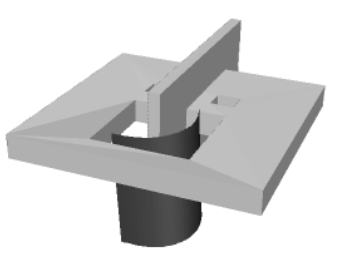

(3)

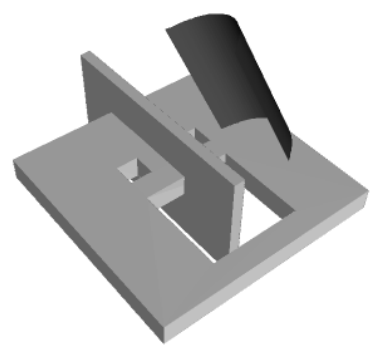

(5)

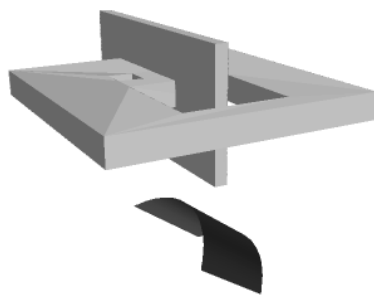

$(2)$

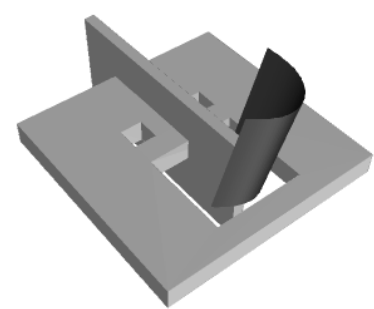

(4)

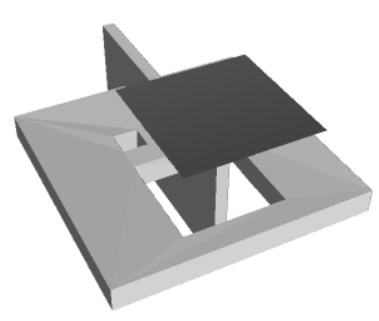

(6)
Figure 7: Experiment with a surface that can only bend.

Subsequently, if the deformations of $c_{1}$ and $c_{2}$ are different, a linear interpolation on the parameter $d$ is used to obtain deformation $c_{2}$ from that of $c_{1}$. Intermediate deformations are read from our precomputed array.

\subsection{Experimental results}

Our code is written in $\mathrm{C}++$ and all the timing results reported here were obtained on an SGI R10000. Our implementation follows closely the description in Section 4 with the exception that enhancement is started after a few basic steps; the number of nodes generated in each basic step is small and our heuristic scheme for enhancement is not very efficient with small roadmaps. 
Figure 7 shows snapshots along a path computed by f-PRM for a 10 control point Bézier curve. The parameters for the basic step of f-PRM are $N=100$, $M=100$, and $K=18$. We assume that we are treating a metal sheet plate and we bound $d_{\text {min }}$ to reflect its elasticity limits. During enhancement the random walk consists of a maximum of 10 reflections, each of which can be 100 steps long. We run f-PRM 10 different times changing the value of the random seed generator. The planner reliably solved the problem all 10 times with an average running time of $3.3 \mathrm{~min}$. Note that we solve a considerably difficult problem. The polyhedral obstacle which obstructs the rectangular hole of the workspace in Figure 7 extends beyond the hole (from below and above). This fact restricts the deformations with which the surface can go through the hole. Another example is shown in Figure 1. Without changing any of the parameters of our planner we can solve that problem with an average running time of $1.2 \mathrm{~min}$. The precomputation of the array of deformations took 22 $\min$.

\section{Planning in the Absence of Manipu- lation Constraints}

We now discuss the application of f-PRM to a case with less restrictive manipulation constraints and energy model. In particular we assume that each control point of the Bézier surface patch is free to move by itself and the energy model simply disallows extensive shear and bending of the surface. We use this example to illustrate the capabilities of our framework by solving high dimensional planning problems. A detailed discussion can be found in [18]. Note that this line of work may be relevant in computer generated animation. The energy model can be used to make the motion look "natural" and the specified constraints would typically be different from manipulation constraints.

\subsection{Implementation choices}

We use a $3 \times 3$ Bézier surface patch for our experiments. To specify a deformation, we need to specify the coordinates of the control points of the surface. Thus this problem has 27 dof. We describe below those implementation choices that are different from the ones of the elastic surface that can only bend.

Energy model: the energy model excludes deformations that correspond to unreasonable bending, extension, or shear of the surface. We divide the energy into three parts $E_{\text {curv }}, E_{\text {area }}, E_{\text {shear }}$ to reflect energy due to curvature, area variation, and shear. These terms are computed on a $10 \times 10$ grid of points sampled evenly across the surface. Let $x_{i j}, i, j=1, \ldots, 10$ be the grid points, and let $x_{i j}^{c_{0}}, i, j=1, \ldots, 10$ be the corresponding grid points at the load-free shape for referring to properties of that shape. Then

$$
\begin{aligned}
E_{\text {curv }} & =\max _{i j}\left\{\sin ^{2}\left(\operatorname{angle}\left(\left(x_{i j}-x_{i j-1}\right),\left(x_{i j+1}-x_{i j}\right)\right)\right)\right\} \\
E_{\text {area }} & =\max _{i}\left\{\sum_{j}\left|x_{i j}-x_{i j+1}\right|-\sum_{j}\left|x_{i j}^{c_{0}}-x_{i j+1}^{c_{0}}\right|\right\} \\
E_{\text {shear }} & =\max _{i j}\left\{\cos ^{2}\left(\operatorname{angle}\left(\left(x_{i+1}-x_{i j}\right),\left(x_{i j+1}-x_{i j}\right)\right)\right)\right\} .
\end{aligned}
$$

Once the energy is computed, each component is compared to a predefined cutoff value.

Generation of random configurations: a deformation is specified by the position of each control point $P_{i j}$. To avoid generating huge deformations, we displace each of the $P_{i j}$ 's of the load free configuration by a vector whose magnitude is bounded and whose orientation is chosen at random. This idea is explored further in [18]. Again, random rigid transformations can be applied to a deformation to obtain random configurations.

Local planner: the local planner simultaneously interpolates the rigid transformations and deformations of the corresponding configurations. For the deformation, the position of each control point is interpolated in $\mathbf{R}^{3}$.

\subsection{Experimental results}

We run f-PRM 10 different times changing the value of the random seed generator for the workspace of Figure 8 . The workspace and surface dimensions are the same as the ones used in Section 5.2. The parameters of 


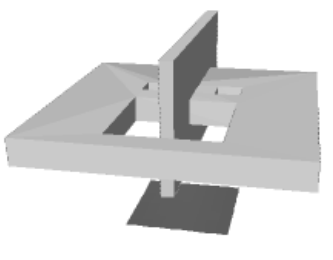

(1)

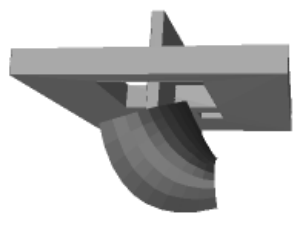

(3)

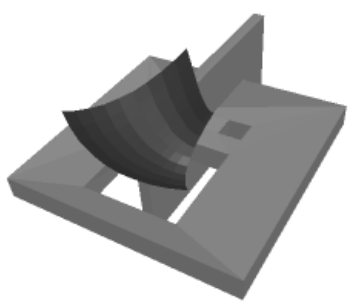

(5)

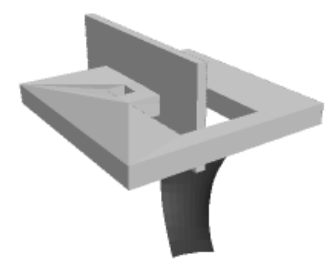

$(2)$

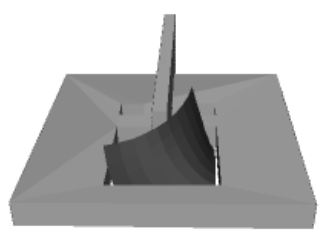

(4)

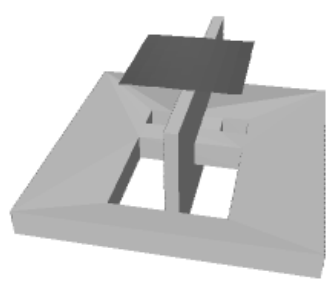

(6)
Figure 8: Snapshots along a path computed by f-PRM.

f-PRM are kept the same as in Section 5.2. The planner reliably solved the unconstrained problem all 10 times with an average time of 4.7 hours. Note that we now solve a 27-dof problem so we expect to have much higher running times than those of Section 5.2. Keeping the parameters of the planner the same, we find that the average running time for solving the problem of Figure 1 is $9.1 \mathrm{~min}$, further emphasizing the difficulty of the example in Figure 8.

\section{Discussion}

This paper investigates the problem of path planning for a thin elastic metal plate. We used the Bézier rep- resentation for describing the geometry of the plate and provided a model for its elastic energy. We compute the shape of the plate with respect to a set of grasping constraints by minimizing the elastic energy of the deformation. A probabilistic roadmap approach is developed to compute paths for the plate. Our experiments show that our new planner, f-PRM, can successfully solve high-dimensional problems, but can also deal with highly constrained and realistic models when the deformation is controlled by manipulation constraints. We also extended our framework to the case of less restrictive energy models and manipulation constraints that typically require solving higher dimensional planning problems.

Our work is a first step in the direction of considering object flexibility in general, and elasticity in particular, during planning. It raises a number of interesting issues which we expect to address in the future. These include: studying different geometric representations for the flexible objects, allowing for more complex manipulation constraints, developing efficient strategies for planning local paths, and devising methods that smooth computed paths or possibly relax the energy of the object along these paths.

\section{Acknowledgments}

Lydia Kavraki and Florent Lamiraux are partially supported by NSF CAREER Award IRI-970228. Christopher Holleman is supported on RUE IRI-970228. The authors would like to thank Joe Warren for many helpful discussions and creative ideas for this work, and Jean-Claude Latombe and Leo Guibas for their comments. The comments of the anonymous referees were also very helpful to improve the presentation of this paper.

\section{References}

[1] J. M. Ahuactzin, E.-G. Talbi, P. Bessière, and E. Mazer. Using genetic algorithms for robot motion planning. In 10th Europ. Conf. Artific. Intelligence, pages 671-675, London, England, 1992.

[2] N. Amato, O. Burchan Bayazit, L. Dale, C. Jones, D. Vallejo, "OBPRM: an obstacle-based PRM for 3D 
Towards Planning for Elastic Objects

Workspaces", Preprints of the 3rd Workshop on the alg. found. of Rob., Houston, TX, 1998.

[3] D. Baraff and A. Witkin. Dynamic simulation of nonpenetrating rigid bodies. Computer Graphics (SIGGRAPH'92), pages 303-308, 1992.

[4] J. Barraquand and J.-C. Latombe. Robot motion planning: A distributed representation approach. Int. J. of Robotics Research, 10:628-649, 1991.

[5] R. Beach. An introduction to the Curves and Surfaces of Computer-Aided Design. Van Nostrand-Reinhold, NY, 1991.

[6] S. Borg. Fundamentals of Engineering Elasticity. World Scientific Publishing Company, Singapore, 1990.

[7] J. Burdick, J. Radford, and G. Chirikjian. A sidewinding locomotion gait for hyper-redundant robots. In Proc. IEEE Int. Conf. on Rob. and Autom., pages 101-106, Atlanta, GA, 1993.

[8] G. Celniker and D. Gossard. Deformable curve and surface finite-elements for free-form shape design. Computer Graphics (SIGGRAPH'91), pages 257-266, 1991.

[9] H. Chang. Personal Communication.

[10] H. Chang and T. Li. Assembly maintainability study with motion planning. In Proc. IEEE Int. Conf. on Rob. and Autom., pages 1012-1019, 1995.

[11] P. Chen and Y. Hwang. Sandros: A motion planner with performance proportional to task difficulty. In Proc. of IEEE Int. Conf. Robotics and Automation, pages 2346-2353, Nice, France, 1992.

[12] B. Donald and P. Xavier. Provably good approximation algorithms for optimal kinodynamic planning: Robots with decoupled dynamics bounds. Algorithmica, 14:443-479, 1995.

[13] B. Donald, P. Xavier, J. Canny, and J. Reif. Kinodynamic motion planning. J. of the ACM, 40:1048-1066, 1993.

[14] G. Farin. Curves and Surfaces for Computer-Aided Geometric Design. Academic Press, San Diego, 1988.

[15] K. Goldberg, D. Halperin, J.-C. Latombe, and R. Wilson, editors. Algorithmic Foundations of Robotics. A K Peters, Ltd., 1995.
[16] K. Gupta and X. Zhu. Practical motion planning for many degrees of freedom: A novel approach within sequential framework. In Proc. of IEEE Int. Conf. Robotics and Automation, pages 2038-2043, San Diego, CA, 1994.

[17] D. Halperin, L. Kavraki, and J.-C. Latombe. Robotics. In J. Goodman and J. O'Rourke, editors, Discrete and Computational Geometry, pages 755-778. CRC Press, NY, 1997.

[18] C. Holleman, L. Kavraki, and J. Warren. Planning paths for a flexible surface patch. In Proc. IEEE Int. Conf. Robotics and Automation, 1998.

[19] L. Kavraki and J.-C. Latombe. Randomized preprocessing of configuration space for fast path planning. In Proc. IEEE Int. Conf. Robotics and Automation, pages 2138-2145, San Diego, CA, 1994.

[20] L. Kavraki, P. Svestka, J.-C. Latombe, and M. Overmars. Probabilistic roadmaps for fast path planning in high dimensional configuration spaces. IEEE Tr. on Rob. and Autom., 12:566-580, 1996.

[21] J.-C. Latombe. Robot Motion Planning. Kluwer, Boston, MA, 1991.

[22] J.-P. Laumond. Feasible trajectories for mobile robots with kinematic and environment constraints. In Proc. Intelligent Autonomous Systems, pages 346-354, 1987.

[23] J.-P. Laumond, P. Jacobs, M. Taix, and R. Murray. A motion planner for nonholonomic mobile robots. IEEE Tr. on Rob. and Autom., 10:577-593, 1994.

[24] M. Lin, D. Manocha, J. Cohen, and S. Gottschalk. Collision detection: Algorithms and applications. In Goldberg et al. [15], pages 129-141.

[25] H. Nakagaki and K. Kitagaki. Study of deformation tasks of a flexible wire. In Proc. IEEE Int. Conf. on Rob. and Autom., Albuquerque, NM, 1997.

[26] W. Ngugen and J. Mills. Multi-robot control for flexible fixtureless assembly of flexible sheet metal auto body parts. In Proc. IEEE Int. Conf. on Rob. and Autom., pages 2340-2345, Minneapolis, MN, 1996.

[27] J. Ostrowski and J. Burdick. Gait kinematics for a serpentine robot. In Proc. IEEE Int. Conf. on Rob. and Autom., pages 1294-1299, Minneapolis, MN, 1996.

[28] M. Overmars and P. Svestka. A probabilistic learning approach to motion planning. In Goldberg et al. [15], pages $19-37$. 
[29] W. H. Press, S. A. Teutolsky, W. T. Vetterling, and B. P. Flannery. Numerical Recipes in C. Cambridge University Press, 1995.

[30] M. Spong and M. Viyasagar. Robot Dynamics and Control. John Wiley and Sons, NY, 1989.

[31] D. Sun, X. Shi, and Y. Liu. Modeling and cooperation of two-arm robotic system manipulating a deformable object. In Proc. IEEE Int. Conf. on Rob. and Autom., pages 2346-2351, Albuquerque, NM, 1996.

[32] M. Surles. An algorithm with linear complexity for interactive physically-based modeling of large proteins. Computer Graphics, 26:221-230, 1992.

[33] P. Svestka and M. Overmars. Motion planning for carlike robots using a probabilistic learning approach. Int. J. of Robotics Research, 16(2):119-143, 1997.

[34] D. Terzopoulos, J. Platt, A. Barr, and K. Fleischer. Elastically deformable models. Computer Graphics (SIGGRAPH'87), 21(4):205-214, 1987.

[35] D. Terzopoulos and A. Witkin. Physically based models with rigid and deformable components. IEEE Computer Graphics and Applications, pages 41-51, November 1988 .

[36] D. Terzopoulos, A. Witkin, and M. Kass. Energy constraints on deformable models: recovering shape and non-rigid motion. In Proc. of AAAI 87, Seattle, 1987.

[37] J. Thingvold and E. Cohen. Physical modeling with b-spline surfaces for interactive design and animation. Computer Graphics (SIGGRAPH'90), 24(2):129-139, 1990.

[38] G. Wempner. Mechanics of Solids with applications to thin bodies. McGraw-Hill, NY, 1991.

[39] R. H. Wilson. On Geometric Assembly Planning. PhD thesis, Stanford Univ., March 1992. Stanford Technical Report STAN-CS-92-1416.

[40] A. Witkin and W. Welch. Fast animation and control of non-rigid structures. Computer Graphics (SIGGRAPH'90), pages 243-252, 1990.

\section{Appendix: Elasticity of a Plate}

Elasticity has been studied extensively in mechanics $[6,38]$. This theory is based on the existence of a potential energy function per unit of volume which depends on the temperature and deformation fields within the material. An elastic body can be deformed by external causes such as grasping which imposes constraints on the position of a subset of points of the body. The deformation field induced minimizes the elastic potential energy over the whole body.

For materials such as metals, the deformation field has to remain small to ensure the reversibility of the deformation. In this case, the linear elastic model is generally used. This model asserts that the elastic energy is quadratic with respect to the strain field of a deformation.

In the case of a thin plate, the elastic energy can be approximated by the deformation of the medium surface of the plate, ignoring for a moment the thickness of the plate. Then, the strain field, in analogy with the strain vector in the $1 \mathrm{D}$ case (see Section 3.3), has 3 components measuring planar strain and 3 components measuring curvature. Let us parameterize the undeformed surface as follows

$$
S_{0}\left(x_{1}, x_{2}\right)=x_{1} \vec{w}_{1}+x_{2} \vec{w}_{2}
$$

where $\left(\vec{w}_{1}, \vec{w}_{2}, \vec{w}_{3}\right)$ is an orthonormal basis. Let us also consider a deformation for which the point of coordinates $\left(x_{1}, x_{2}\right)$ is transported to $S\left(x_{1}, x_{2}\right)$. The temperature is assumed to be constant. All the computations needed to establish the results below can be found in [38].

\section{A. Strain field of the plate}

The strain field of a deformation of a surface is defined, at each point of the surface, by six coefficients: three of these coefficients account for planar deformation while three account for curvature.

Planar strain coefficients The planar strain coefficients are computed by comparing the tangent vectors to the undeformed surface with those of the deformed one. Let us use the following notation for these tangent vectors

$$
\begin{aligned}
& t_{1}\left(x_{1}, x_{2}\right)=\frac{\partial S\left(x_{1}, x_{2}\right)}{\partial x_{1}} \\
& t_{2}\left(x_{1}, x_{2}\right)=\frac{\partial S\left(x_{1}, x_{2}\right)}{\partial x_{2}}
\end{aligned}
$$


Towards Planning for Elastic Objects

$$
\begin{aligned}
t_{1}^{0}\left(x_{1}, x_{2}\right) & =\frac{\partial S_{0}\left(x_{1}, x_{2}\right)}{\partial x_{1}} \\
t_{2}^{0}\left(x_{1}, x_{2}\right) & =\frac{\partial S_{0}\left(x_{1}, x_{2}\right)}{\partial x_{2}} .
\end{aligned}
$$

The strain coefficients correspond to the difference between the inner products of the tangent vectors before and after deformation, as follows

$$
\begin{aligned}
& e_{11}=\frac{1}{2}\left(t_{1} \cdot t_{1}-t_{1}^{0} \cdot t_{1}^{0}\right)=\frac{1}{2}\left(t_{1} \cdot t_{1}-1\right) \\
& e_{22}=\frac{1}{2}\left(t_{2} \cdot t_{2}-t_{2}^{0} \cdot t_{2}^{0}\right)=\frac{1}{2}\left(t_{2} \cdot t_{2}-1\right) \\
& e_{12}=\frac{1}{2}\left(t_{1} \cdot t_{2}-t_{1}^{0} \cdot t_{2}^{0}\right)=\frac{1}{2}\left(t_{1} \cdot t_{2}\right) .
\end{aligned}
$$

$e_{11}$ and $e_{22}$ are called the extension coefficients in the $x_{1}$ and $x_{2}$ directions, whereas $e_{12}$ is the shear between these directions.

Notice that the coefficient $\varepsilon$ of the one-dimensional deformation of Section 3 is a first order approximation of the real extension coefficient in the $u$ direction.

Curvature coefficients To express the curvature, we need the normalized tangent and normal vectors to the deformed surface

$$
\begin{aligned}
\bar{t}_{1} & =t_{1} /\left\|t_{1}\right\| \\
\bar{t}_{2} & =t_{2} /\left\|t_{2}\right\| \\
n & =\left(\bar{t}_{1} \times \bar{t}_{2}\right) /\left\|\bar{t}_{1} \times \bar{t}_{2}\right\| .
\end{aligned}
$$

The curvature of the deformed surface can be represented by the three following coefficients

$$
\begin{aligned}
\chi_{11} & =\frac{1}{\left\|t_{1}\right\|} \frac{\partial \bar{t}_{1}}{\partial d x_{1}} \cdot n \\
\chi_{22} & =\frac{1}{\left\|t_{2}\right\|} \frac{\partial \bar{t}_{2}}{\partial d x_{2}} \cdot n \\
\chi_{12} & =\frac{1}{\left\|t_{1}\right\|} \frac{\partial \bar{t}_{2}}{\partial d x_{1}} \cdot n=\frac{1}{\left\|t_{2}\right\|} \frac{\partial \bar{t}_{1}}{\partial d x_{2}} \cdot n .
\end{aligned}
$$

\section{B. Elastic energy of the plate}

We can group the coefficients defined above in two matrices to simplify the expression of the elastic energy

$$
e_{s}=\left(\begin{array}{ll}
e_{11} & e_{12} \\
e_{12} & e_{22}
\end{array}\right), \quad \chi=\left(\begin{array}{ll}
\chi_{11} & \chi_{12} \\
\chi_{12} & \chi_{22}
\end{array}\right) .
$$

These matrices represent in fact the planar strain tensor and the curvature tensor. With this notation, the elastic energy is the sum of two terms:

$$
\psi_{s}\left(e_{s}, \chi\right)=\psi_{s}^{e}\left(e_{s}\right)+\psi_{s}^{\chi}(\chi),
$$

with

$$
\psi_{s}^{e}\left(e_{s}\right)=\frac{E h}{2} \frac{(1-\nu) \operatorname{tr}\left(e_{s}^{2}\right)+\nu\left(\operatorname{tr} e_{s}\right)^{2}}{(1-\nu)(1+\nu)}
$$

and

$$
\psi_{s}^{\chi}(\chi)=\frac{E h^{3}}{24} \frac{(1-\nu) \operatorname{tr}\left(\chi^{2}\right)+\nu(\operatorname{tr} \chi)^{2}}{(1-\nu)(1+\nu)} .
$$

The elastic energy is thus quadratic with respect to the strain field. Moreover, we can see from the last two equations that since $h$ is assumed to be small, the energy deformation due to the curvature is far smaller than the energy due to extension and shear within the tangent plane of the surface. This fact explains why it is easier to bend a thin plate than to extend it. 